\title{
Bethe-Heitler cascades as a plausible origin of hard spectra in distant TeV blazars
}

\author{
Y. G. Zheng ${ }^{1}$, C. Y. Yang ${ }^{2,3}$, and S. J. Kang ${ }^{4}$ \\ ${ }^{1}$ Department of Physics, Yunnan Normal University, 650092 Kunming, PR China \\ e-mail: ynzyg@yun.edu.cn \\ 2 Yunnan Observatories, Chinese Academy of Sciences, 650011 Kunming, PR China \\ 3 Key Laboratory for the Structure and Evolution of Celestial Objects, Chinese Academy of Sciences, 100049 Beijing, PR China \\ ${ }^{4}$ Department of Physics and Electronics Science, Liupanshui Normal University, Liupanshui, 553004 Guizhou, PR China
}

Received 9 June 2015 / Accepted 30 September 2015

\section{ABSTRACT}

\begin{abstract}
Context. Very high-energy (VHE) $\gamma$-ray measurements of distant TeV blazars can be nicely explained by TeV spectra induced by ultra high-energy cosmic rays.

Aims. We develop a model for a plausible origin of hard spectra in distant TeV blazars.

Methods. In the model, the TeV emission in distant $\mathrm{TeV}$ blazars is dominated by two mixed components. The first is the internal component with the photon energy around $1 \mathrm{TeV}$ produced by inverse Compton scattering of the relativistic electrons on the synchrotron photons (SSC) with a correction for extragalactic background light absorbtion and the other is the external component with the photon energy more than $1 \mathrm{TeV}$ produced by the cascade emission from high-energy protons propagating through intergalactic space.

Results. Assuming suitable model parameters, we apply the model to observed spectra of distant TeV blazars of 1ES $0229+200$. Our results show that 1) the observed spectrum properties of 1ES $0229+200$, especially the TeV $\gamma$-ray tail of the observed spectra, could be reproduced in our model and 2) an expected TeV $\gamma$-ray spectrum with photon energy $>1 \mathrm{TeV}$ of $1 \mathrm{ES} 0229+200$ should be comparable with the 50-h sensitivity goal of the Cherenkov Telescope Array (CTA) and the differential sensitivity curve for the one-year observation with the Large High Altitude Air Shower Observatory (LHAASO).

Conclusions. We argue that strong evidence for the Bethe-Heitler cascades along the line of sight as a plausible origin of hard spectra in distant TeV blazars could be obtained from VHE observations with CTA, LHAASO, HAWC, and HiSCORE.
\end{abstract}

Key words. BL Lacertae objects: individual: 1ES 0229+200 - radiation mechanisms: non-thermal

\section{Introduction}

A blazar is a special class of active galactic nucleus (AGN) with a non-thermal continuum emission that arises from the jet emission taking place in an AGN whose jet axis is closely aligned with the observer's line of sight (Urry \& Padovani 1995). Blazars are dominated by rapid and large amplitude variability (e.g., Raiteri et al. 2012; Sobolewska et al. 2014). Multiwavelength observations show that their broad spectral energy distributions (SED) from the radio to the $\gamma$-rays bands generally exhibits two humps, indicating two components. It is generally accepted that the low-energy component that extends from radio up to ultraviolet, or in some extreme cases to a few keV X-rays (Costamante et al. 2001), is produced by synchrotron radiation from relativistic electrons in the jet (Urry 1998), though the origin of the high-energy component that covers the X-ray and $\gamma$-ray energy regime remains an open issue. There are two kinds of theoretical models describing the highenergy photon emission in these blazars, the leptonic and the hadronic model. In the leptonic model scenarios, the high-energy component is probably produced from inverse Compton (IC) scattering of the relativistic electrons either on the synchrotron photons (e.g., Maraschi et al. 1992; Bloom \& Marscher 1996; Mastichiadis \& Kirk 1997; Konopelko et al. 2003) and/or on some other photon populations (e.g., Dermer et al. 1992; Dermer \& Schlickeiser. 1993; Sikora et al. 1994; Ghisellini \& Madau 1996; Böttcher \& Dermer 1998). In contrast, the hadronic model argues that high-energy $\gamma$ rays are produced by either proton synchrotron radiation in high enough magnetic fields (Aharonian 2000; Mücke \& Protheroe 2001; Mücke et al. 2003; Petropoulou 2014), or mesons and leptons through the cascade initiated by proton-proton or proton-photon interactions (e.g., Mannheim \& Biermann 1992; Mannheim 1993; Pohl \& Schlickeiser 2000; Atoyan \& Dermer 2001).

The imaging atmospheric Cherenkov Telescopes (IACTs) have so far detected about 50 very high-energy (VHE; $E_{\gamma}>$ $100 \mathrm{GeV}) \gamma$-ray blazars with redshifts up to $z \sim 0.6^{1}$. It is believed that the primary $\mathrm{TeV}$ photons propagating through intergalactic space should be attenuated due to their interactions with the extragalactic background light (EBL) to produce electronpositron $\left(e^{ \pm}\right)$pairs (e.g., Nikishov 1962; Gould \& Schreder 1966; Stecker et al. 1992; Ackermann et al. 2012; Abramowski et al. 2013; Dwek \& Krennrich 2013; Sanchez et al. 2013). However, the observed spectra from distant blazars do not show a sharp cutoff at energies around $1 \mathrm{TeV}$, which would be expected from simple $\gamma$-ray emission models with a correction for EBL absorbtion (e.g., Stecker et al. 2006; Aharonian et al. 2006a; Costamante et al. 2008; Acciari et al. 2009; Abramowski et al. 2012). Excluding a large uncertainty in the measured redshifts and in the spectral indices (Costamante 2013) and excluding the lower levels of EBL (Aharonian et al. 2006b; Mazin \& Raue 2007; Finke \& Razzaque 2009), the observed

http://tevcat.uchicago.edu 
spectral hardening assumes either that there are axion-like particles (de Angelis et al. 2007; Simet et al. 2008; Sanchez-Conde et al. 2009) or a Lorentz invariance violation (Kifune 1999; Protheroe \& Meyer 2000). Alternatively, now that the AGN jets are believed to be one of the most powerful sources of cosmic rays, as long as the intergalactic magnetic fields (IGMF) deep in the voids are less than a femtogauss, the point images of distant blazars, which are produced by the interaction of the energy protons with the background photons along the line of sight, should be observed by IACTs (Essey et al. 2011a). In this scenario, the hard $\mathrm{TeV}$ spectra can be produced by the cascade emission from high-energy protons propagating through intergalactic space (Essey \& Kusenko 2010; Essey et al. 2010; 2011b; Razzaque et al. 2012; Aharonian et al. 2013; Takami et al. 2013; Zheng \& Kang 2013).

In this paper, we study the possible $\mathrm{TeV}$ emission in distant $\mathrm{TeV}$ blazars. We argue that the $\mathrm{TeV}$ emission in distant $\mathrm{TeV}$ blazars is dominated by two components, the internal component with the photon energy around $1 \mathrm{TeV}$ produced by IC scattering of the relativistic electrons on the synchrotron photons (SSC) with a correction for EBL absorbtion and the external component with the photon energy of more than $1 \mathrm{TeV}$ produced by the cascade emission from high-energy protons propagating through intergalactic space. Generally, the external photons are generated in two types photohadronic interactions process along the line of sight. In the first, the proton interaction with cosmic microwave background (CMB) photons would produce $e^{ \pm}$pairs, and the pairs would give rise to electromagnetic cascades. This process is called the Bethe-Heitler pair production ( $p e$ ) process. In the second process, the proton interaction with EBL photons would produce pions, and the pion decay accompanying the photons. This process is called the photopion production $(p \pi)$ process. Although the pe process contribution to the production of secondary photons is illustrated at the source (Dimitrakoudis et al. 2012; Murase 2012; Murase et al. 2012; Petropoulou 2014; Petropoulou \& Mastichiadis 2015), the high-energy astrophysical interest focuses on the $p \pi$ process along the line of sight because the pe process is not associated with any neutrinos and neutrons (e.g., Inoue et al. 2013; Kalashev et al. 2013). The aim of the present work is to study in more detail the contribution of pairs injected by the pe process along the line of sight to the $\mathrm{TeV}$ spectra in distant blazars.

Throughout the paper, we assume the Hubble constant $H_{0}=$ $75 \mathrm{~km} \mathrm{~s}^{-1} \mathrm{Mpc}^{-1}$, the matter energy density $\Omega_{\mathrm{M}}=0.27$, the radiation energy density $\Omega_{\mathrm{r}}=0$, and the dimensionless cosmological constant $\Omega_{\Lambda}=0.73$.

\section{The model}

We calculate the spectra of the internal photon component within the traditional SSC model frame, and we focus on an overlooked Bethe-Heitler pairs cascade process along the line of sight for the spectra of the external photon component. In the following, we give a brief description of the model for possible $\mathrm{TeV}$ emission in a distant $\mathrm{TeV}$ blazar.

\subsection{Internal photon component}

We assume that a single homogeneous spherical radiation region filled with extreme-relativistic electrons, in which there is a randomly originated homogeneous magnetic field and constant electron number density. We adopt a broken power-law function with a sharp cut-off to describe the electron energy distribution in the radiation region,

$N_{\mathrm{e}, \text { in }}\left(E_{\mathrm{e}, \text { in }}\right)=\left\{\begin{array}{l}K_{0} E_{\mathrm{e}, \text { in }}^{-n_{1}}, E_{\mathrm{e}, \text { in }, \text { min }} \leq E_{\mathrm{e}, \text { in }} \leq E_{\mathrm{e}, \text { in }, \mathrm{b}} \\ K_{1} E_{\mathrm{e}, \text { in }}^{-n_{2}}, E_{\mathrm{e}, \text { in }, \mathrm{b}} \leq E_{\mathrm{e}, \text { in }} \leq E_{\mathrm{e}, \text { in }, \mathrm{cut}}\end{array}\right.$

where $E_{\mathrm{e}, \text { in }}$ is the energy of electron in the internal emission region and $K_{1}=K_{0} E_{\mathrm{e}, \mathrm{in}, \mathrm{b}}^{\left(n_{2}-n_{1}\right)}$. Based on the above electron number density $N_{\mathrm{e}, \text { in }}\left(E_{\mathrm{e}, \text { in }}\right)$, we can calculate the synchrotron intensity $I_{\mathrm{s}}\left(E_{\gamma}\right)$ and the intensity of self-Compton radiation $I_{\mathrm{c}}\left(E_{\gamma}\right)$, and then calculate the intrinsic photon spectrum $\mathrm{d} N_{\gamma}^{\text {int }}\left(E_{\gamma}\right) / \mathrm{d} E_{\gamma}$ at the observer's frame (e.g., Katarzynski et al. 2001; Zheng \& Zhang 2011; Zheng \& Kang 2013). Taking into account the absorption effect, the flux density observed at the Earth becomes

$\frac{\mathrm{d} N_{\gamma}^{\mathrm{in}}\left(E_{\gamma}\right)}{\mathrm{d} E_{\gamma}}=\frac{\mathrm{d} N_{\gamma}^{\mathrm{int}}\left(E_{\gamma}\right)}{\mathrm{d} E_{\gamma}} \exp \left[-\tau\left(E_{\gamma}, z\right)\right]$

where $\tau\left(E_{\gamma}, z\right)$ is the absorption optical depth due to interactions with the EBL (e.g., Kneiske et al. 2004; Dwek \& Krennrich 2005; Franceschini et al. 2008). In our calculation, we use the absorption optical depth which is deduced by the EBL model in Dwek \& Krennrich (2005).

\subsection{External photon component}

Active galactic nuclei are expected to accelerate cosmic rays to energies up to $\sim 10^{11} \mathrm{GeV}$ during rare bursts or flares (Dermer et al. 2009; Murase \& Takami 2009). The high-energy cosmic rays with energies below the Greisen-Zatsepin-Kuzmin (GZK) cutoff of about $5 \times 10^{10} \mathrm{GeV}$ can propagate cosmological distances without significant energy loss (Greisen 1966; Zatsepin \& Kuzmin 1966). However, with a small probability, these protons should interact with the CMB photons and trigger electromagnetic cascades. The present work differs from the earlier studies on which the electromagnetic cascades were modeled using a standard Monte Carlo approach (e.g., Essey et al. 2010). We employ a Bethe-Heitler pair-creation rate in the $\delta$-functional approximation and we concentrate on the protons with energy below the GZK cutoff, which would propagate through cosmological distances.

\subsubsection{Bethe-Heitler pairs production}

We consider an isotropic target photon field with energy $E_{\mathrm{s}}=$ $\epsilon_{\mathrm{s}} m_{\mathrm{e}} c^{2}$ interaction on the proton with energy $E_{\mathrm{p}}=\gamma_{\mathrm{p}} m_{\mathrm{p}} c^{2}$. We let $N_{\mathrm{p}}\left(E_{\mathrm{p}}\right)$ and $N_{\mathrm{ph}}\left(E_{\mathrm{s}}\right)$ be functions characterizing the energy distributions of protons and soft photons and the collision rate in the unit of $\mathrm{s}^{-1}$ can be given by Romero \& Vila (2008)

$w_{\mathrm{p} \gamma, \mathrm{e}}\left(E_{\mathrm{p}}\right)=\frac{m_{\mathrm{p}}^{2} m_{\mathrm{e}}^{2} c^{9}}{2 E_{\mathrm{p}}^{2}} \int_{E_{\mathrm{s}, \min }}^{\infty} \frac{N_{\mathrm{ph}}\left(E_{\mathrm{s}}\right)}{E_{\mathrm{s}}^{2}} \mathrm{~d} E_{\mathrm{s}} \int_{\epsilon_{\mathrm{s}, \min }^{\prime}}^{\epsilon_{\mathrm{s}, \max }^{\prime}} \sigma_{\mathrm{p} \gamma, \mathrm{e}}\left(\epsilon_{\mathrm{s}}^{\prime}\right) \epsilon_{\mathrm{s}}^{\prime} \mathrm{d} \epsilon_{\mathrm{s}}^{\prime}$,

where $\epsilon_{\mathrm{s}}^{\prime}=\gamma_{\mathrm{p}} \epsilon_{\mathrm{s}}\left(1-\beta_{\mathrm{p}} \cos \theta\right)$ is the energy of the photon in the rest frame of the proton with the angle between the proton and photon directions $\theta$, and the proton's velocity $\beta_{\mathrm{p}}$ in units of $c$. Because the Bethe-Heitler pairs production are with a threshold energy of $\sim 1 \mathrm{MeV}$ for the photon in the proton rest frame, we can obtain $\epsilon_{\mathrm{s}, \min }^{\prime}=1 \mathrm{MeV} /\left(m_{\mathrm{e}} c^{2}\right) \sim 2, E_{\mathrm{s}, \min }=1 / 2 \gamma_{\mathrm{p}} \mathrm{MeV}$ (e.g., Mastichiadis \& Kirk 1995). A head-on collision gives the 
maximum energy of the photon in the rest frame of the proton $\epsilon_{\mathrm{s}, \max }^{\prime}=2 \gamma_{\mathrm{p}} \epsilon_{\mathrm{s}}$. The energy distribution of soft photons is described by

$N_{\mathrm{ph}}\left(E_{\mathrm{s}}\right)=\frac{1}{\pi^{2}(\hbar c)^{3}} \frac{E_{\mathrm{s}}^{2}}{\exp \left(E_{\mathrm{s}} / k T\right)-1}$,

where the typical temperature $T=(1+z) 2.73 \mathrm{~K}$ with redshift $z$ and $k$ is the Boltzmann constant. The total cross section $\sigma_{\mathrm{p} \gamma, \mathrm{e}}\left(\epsilon_{\mathrm{s}}^{\prime}\right)$ for which we use the Racah formula as parameterized by Maximon (1968). For $2 \leq \epsilon_{\mathrm{s}}^{\prime} \leq 4$, the expansion is given with a fractional error less than $1.1 \times 10^{-3}$ (Maximon 1968),

$$
\begin{aligned}
\sigma_{\mathrm{p} \gamma \mathrm{e}}\left(\epsilon_{\mathrm{s}}^{\prime}\right) \simeq & \frac{2 \pi}{3} \alpha_{\mathrm{f}} r_{0}^{2} Z^{2}\left(\frac{\epsilon_{\mathrm{s}}^{\prime}-2}{\epsilon_{\mathrm{s}}^{\prime}}\right)^{2} \\
& \times\left(1+\frac{1}{2} \eta+\frac{23}{40} \eta^{2}+\frac{37}{120} \eta^{3}+\frac{61}{192} \eta^{4}\right),
\end{aligned}
$$

where $\eta=\left(\epsilon_{\mathrm{s}}^{\prime}-2\right) /\left(\epsilon_{\mathrm{s}}^{\prime}+2\right), \alpha_{\mathrm{f}}$ is the fine structure constant, $r_{0}$ is the classical electron radius, and $Z$ is the Atomic number of the target atom. In the larger photon energy regime, $\epsilon_{\mathrm{s}}^{\prime} \geq 4$, the expansion can be written with a fractional error less than $4.4 \times 10^{-5}$ as (e.g., Chodorowski et al. 1992)

$$
\begin{aligned}
\sigma_{\mathrm{p} \gamma, \mathrm{e}}\left(\epsilon_{\mathrm{s}}^{\prime}\right) \simeq & \alpha_{\mathrm{f}} r_{0}^{2} Z^{2}\left\{\frac{28}{9} \ln 2 \epsilon_{\mathrm{s}}^{\prime}-\frac{218}{27}+\left(\frac{2}{\epsilon_{\mathrm{s}}^{\prime}}\right)^{2}\right. \\
& \times\left[6 \ln 2 \epsilon_{\mathrm{s}}^{\prime}-\frac{7}{2}+\frac{2}{3} \ln ^{3} 2 \epsilon_{\mathrm{s}}^{\prime}-\ln ^{2} 2 \epsilon_{\mathrm{s}}^{\prime}-\frac{1}{3} \pi^{2} \ln 2 \epsilon_{\mathrm{s}}^{\prime}\right. \\
& \left.+2 \zeta(3)+\frac{\pi^{2}}{6}\right]-\left(\frac{2}{\epsilon_{\mathrm{s}}^{\prime}}\right)^{4}\left(\frac{3}{16} \ln 2 \epsilon_{\mathrm{s}}^{\prime}+\frac{1}{8}\right) \\
& \left.-\left(\frac{2}{\epsilon_{\mathrm{s}}^{\prime}}\right)^{6}\left(\frac{9}{9 \times 256} \ln 2 \epsilon_{\mathrm{s}}^{\prime}-\frac{77}{27 \times 512}\right)\right\}
\end{aligned}
$$

where $\zeta(3) \simeq 1.20206$. Now the Monte Carlo simulation shows that the mean inelasticity can be approximated by its values at the threshold $K_{\mathrm{p} \gamma, \mathrm{e}}\left(\epsilon_{\mathrm{s}}^{\prime}\right)=2 m_{\mathrm{e}} / m_{\mathrm{p}}$ (Mastichiadis et al. 2005). Therefore, the Bethe-Heitler pair-creation rate in the $\delta$-functional approximation is then given (Romero \& Vila 2008) as

$$
\begin{aligned}
Q_{\mathrm{e}}\left(E_{\mathrm{p} \gamma, \mathrm{e}}\right) & =2 \int_{E_{\mathrm{p}, \min }}^{E_{\mathrm{p}, \max }} N_{\mathrm{p}}\left(E_{\mathrm{p}}\right) w_{\mathrm{p} \gamma, \mathrm{e}}\left(E_{\mathrm{p}}\right) \delta\left(E_{\mathrm{p} \gamma, \mathrm{e}}-\frac{m_{\mathrm{e}}}{m_{\mathrm{p}}} E_{\mathrm{p}}\right) \mathrm{d} E_{\mathrm{p}} \\
& =2 \frac{m_{\mathrm{p}}}{m_{\mathrm{e}}} N_{\mathrm{p}}\left(\frac{m_{\mathrm{p}}}{m_{\mathrm{e}}} E_{\mathrm{p} \gamma, \mathrm{e}}\right) w_{\mathrm{p} \gamma, \mathrm{e}}\left(\frac{m_{\mathrm{p}}}{m_{\mathrm{e}}} E_{\mathrm{p} \gamma, \mathrm{e}}\right) .
\end{aligned}
$$

We parameterize the proton injection spectrum by a constant power-law exponent $\alpha$ and maximum energy $E_{\mathrm{p}, \max }$ :

$$
N_{\mathrm{p}}\left(E_{\mathrm{p}}\right)=N_{0} E_{\mathrm{p}}^{-\alpha} \exp \left(-\frac{E_{\mathrm{p}}}{E_{\mathrm{p}, \text { max }}}\right), E_{\mathrm{p}, \text { min }} \leq E_{\mathrm{p}} \leq E_{\mathrm{p}, \text { max }} .
$$

Where the normalization coefficient $N_{0}$ is determined from the jet power $L_{\mathrm{p}}$ of the protons. The observed data on the most distant sources shows that an effective luminosity of a single AGN in cosmic rays above $10^{7} \mathrm{GeV}$ was in the range $L_{\mathrm{eff}} \sim$ $10^{47}-10^{49} \mathrm{erg} \mathrm{s}^{-1}$. Since the VLBI observation shows that the value of the Doppler factor $\delta$ is less than 10 (Wu et al. 2007), the flux can be beamed with the beaming factor $f_{\text {beam }} \sim 10-10^{3}$, which increases the flux of protons from a blazar with a jet pointing in the direction of Earth (Essey \& Kusenko 2010)

$L_{\mathrm{eff}} \sim 10^{2} f_{\mathrm{p}} \times\left(\frac{f_{\text {beam }}}{100}\right) L_{\mathrm{p}}$

where $f_{\mathrm{p}} \leq 1$ is the fraction of protons.

\subsubsection{Inverse Compton scattering from Bethe-Heitler pairs}

Assuming that the energy of the Bethe-Heitler pairs is lost through interaction with the $\mathrm{CMB}$ photons at the cosmological distance $D$, we can deduce energy distribution of the secondary electrons or positrons from the well-known energy distribution equation

$\frac{\mathrm{d} N_{\mathrm{e}, \mathrm{ex}}\left(E_{\mathrm{e}, \mathrm{ex}}\right)}{\mathrm{d} E_{\mathrm{e}, \mathrm{ex}}}=\left(\frac{\mathrm{d} E_{\mathrm{e}, \mathrm{ex}}}{\mathrm{d} t}\right)^{-1} \int_{E_{\mathrm{e}, \mathrm{ex}}}^{E_{\mathrm{p} \gamma, \mathrm{e}, \max }} Q_{\mathrm{e}}\left(E_{\mathrm{p} \gamma, \mathrm{e}}\right) \mathrm{d} E_{\mathrm{p} \gamma, \mathrm{e}}$,

where $\mathrm{d} E_{\mathrm{e}, \mathrm{ex}} / \mathrm{d} t=4 \sigma_{\mathrm{T}} E_{\mathrm{e}, \mathrm{ex}}^{2} /\left(3 m_{\mathrm{e}}^{2} c^{3} u_{\mathrm{CMB}}\right)$ is the Bethe-Heitler pairs energy loss rates with Thomson cross section $\sigma_{\mathrm{T}}$ and CMB energy density $u_{\mathrm{CMB}}$. Then, the photon spectrum of the IC scattering is given by

$\frac{\mathrm{d} N_{\gamma}^{\mathrm{ex}}\left(E_{\gamma}\right)}{\mathrm{d} E_{\gamma}}=\int_{E_{\mathrm{e}, \mathrm{ex}, \text { min }}}^{\infty} \frac{\mathrm{d} N_{\mathrm{e}, \mathrm{ex}}\left(E_{\mathrm{e}, \mathrm{ex}}\right)}{\mathrm{d} E_{\mathrm{e}, \mathrm{ex}}}\left[\frac{\mathrm{d}^{2} N_{\mathrm{ph}}\left(E_{\mathrm{s}}\right)}{\mathrm{d} E_{\mathrm{s}} \mathrm{d} t}\right]_{\mathrm{ICS}} \mathrm{d} E_{\mathrm{e}, \mathrm{ex}}$,

with the spectrum of the IC scattered photons per electron (Blumenthal \& Gould 1970)

$$
\begin{aligned}
{\left[\frac{\mathrm{d}^{2} N_{\mathrm{ph}}\left(E_{\mathrm{s}}\right)}{\mathrm{d} E_{\mathrm{s}} \mathrm{d} t}\right]_{\mathrm{ICS}}=} & \int_{E_{\mathrm{s}, \min }}^{\infty} \frac{3 \sigma_{\mathrm{T}} m_{\mathrm{e}}^{2} c^{5}}{4 E_{\mathrm{e}, \mathrm{ex}}^{2}} \frac{N_{\mathrm{ph}}\left(E_{\mathrm{s}}\right)}{E_{\mathrm{S}}} \mathrm{d} E_{\mathrm{s}} \times[2 q \ln q \\
& \left.+(1+2 q)(1-q)+\frac{(\Gamma q)^{2}(1-q)}{2(1+\Gamma q)}\right],
\end{aligned}
$$

where $q=E_{1} / \Gamma\left(1-E_{1}\right), \Gamma=4 \epsilon_{\mathrm{s}} E_{\mathrm{e}, \mathrm{ex}} / m_{\mathrm{e}} c^{2}$, and $E_{1}=E_{\gamma} / E_{\mathrm{e}, \mathrm{ex}}$.

Therefore, the $\mathrm{TeV}$ photon spectrum that is observed at the Earth is given by

$$
\frac{\mathrm{d} N_{\gamma}\left(E_{\gamma}\right)}{\mathrm{d} E_{\gamma}}=\frac{\mathrm{d} N_{\gamma}^{\mathrm{in}}\left(E_{\gamma}\right)}{\mathrm{d} E_{\gamma}}+\frac{\mathrm{d} N_{\gamma}^{\mathrm{ex}}\left(E_{\gamma}\right)}{\mathrm{d} E_{\gamma}} .
$$

\section{Constraint on the energy of protons}

The above scenario depends on the energy of protons. Since the IGMF would cause proton or electron deflection in the voids, and the energetic $\gamma$ rays in the EBL photon fields should be absorbed, we can determine the ranges of a proton's energy through these physical process.

As long as the IGMF $B_{\mathrm{IG}}$ is less than 1 femtogauss, the Cosmic ray protons with energies $E_{\mathrm{p}} \leq E_{\mathrm{GZK}}=5 \times 10^{10} \mathrm{GeV}$ can provide an effective transport of the energy over a cosmological distance toward the observer (Essey et al. 2011a). Then they interact with the CMB photons and initiate electromagnetic cascades. On condition that the deflection broadening of the proton beam $\theta_{\mathrm{p}}$ in IGMFs is less than the point spread function $\overline{\theta(E)}$ of the detector, the resulting secondary photons are observed as arriving from a point source. The deflection angles dependence on the IGMF can be written (Aharonian et al. 2010)

$\theta_{\mathrm{p}} \simeq 0.05 \operatorname{arcmin}\left(\frac{10^{9} \mathrm{GeV}}{E_{\mathrm{p}}}\right)\left(\frac{B_{\mathrm{IG}}}{10^{-15} G}\right)\left(\frac{l_{\mathrm{c}}}{\mathrm{Mpc}} \frac{\mathrm{d}}{\mathrm{Gpc}}\right)^{1 / 2}$,

where $l_{\mathrm{c}}$ is a correlation length of the random fields and $d \sim c z / H_{0}$ is luminosity distance. We consider a VHE blazar with redshift $z$, and the typical point spread function of the HESS telescope array, $\overline{\theta(E)} \simeq 3.0$ arcmin. The constraint condition $\theta_{\mathrm{p}} \leq \overline{\theta(E)}$ could result in $E_{\mathrm{p}} \geq E_{\mathrm{p} \text {,low }}=3.3 \times$ $10^{7} z^{1 / 2}\left(l_{\mathrm{c}} / 1 \mathrm{Mpc}\right)^{1 / 2}\left(B_{\mathrm{IG}} / 10^{-15} \mathrm{G}\right) \mathrm{GeV}$. 


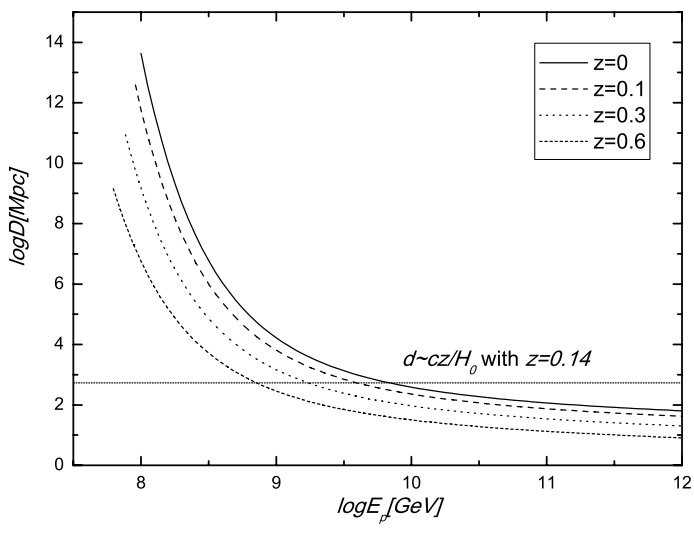

Fig. 1. Secondary emission region as a function of energy and redshift. The distance to the source that is estimated by using Hubble's law with $z=0.14$ is also exhibited as a short dotted line.

Alternatively, VHE $\gamma$-ray photon emission from the region is attenuated by photons from the EBL (e.g., Kneiske et al. 2004; Dwek \& Krennrich 2005). It is well-known that the $\gamma$-ray zone can be defined by the $\gamma$-ray absorption mean free path $\lambda_{\gamma \gamma} \sim 190\left(n_{\mathrm{IR}} / 0.01 \mathrm{~cm}^{-3}\right)^{-1} \mathrm{Mpc}$ with the infrared photon number density $n_{\mathrm{IR}}$ (e.g., Venters 2010). As long as the magnetic field is significantly small, an extremely efficient cascade development could enlarge the $\gamma$-ray zone, typically $\lambda_{\gamma \text {,eff }} \sim 2-3 \lambda_{\gamma \gamma}$ (Aharonian et al. 2013). In this scenario, we argue that if the Bethe-Heitler cascades contribution to the observed hard spectra behavior of blazars is true, the secondary $\gamma$-ray emission region $D$ should satisfy a relation $d-D \leq \lambda_{\gamma \text {,eff }}$. In our work, due to the cascades the electrons are the main origin of the pe process, the adoption $D \simeq \lambda_{\mathrm{p} \gamma, \mathrm{e}}=c / t_{\mathrm{p} \gamma, \mathrm{e}}^{-1}\left(E_{\mathrm{p}}\right)$ is justified. Here,

$$
\begin{aligned}
t_{\mathrm{p} \gamma, \mathrm{e}}^{-1}\left(E_{\mathrm{p}}\right)= & \frac{m_{\mathrm{p}}^{2} m_{\mathrm{e}}^{2} c^{9}}{2 E_{\mathrm{p}}^{2}} \int_{E_{\mathrm{s}, \min }}^{\infty} \frac{N_{\mathrm{ph}}\left(E_{\mathrm{s}}\right)}{E_{\mathrm{s}}^{2}} \mathrm{~d} E_{\mathrm{s}} \\
& \times \int_{\epsilon_{\mathrm{s}, \min }^{\prime}}^{\epsilon_{\mathrm{s}, \max }} K_{\mathrm{p} \gamma, \mathrm{e}}\left(\epsilon_{\mathrm{s}}^{\prime}\right) \sigma_{\mathrm{p} \gamma, \mathrm{e}}\left(\epsilon_{\mathrm{s}}^{\prime}\right) \epsilon_{\mathrm{s}}^{\prime} \mathrm{d} \epsilon_{\mathrm{s}}^{\prime}
\end{aligned}
$$

is the cooling rate of the protons in CMB photon fields (Romero $\&$ Vila 2008). Obviously, in the case of a broad energy distribution of protons, the main contribution to the secondary $\gamma$-ray flux comes from some energetic proton ranges in which the secondary $\gamma$-ray emission region is comparable to the distance of the source, i.e., $D \sim d$. We show the secondary emission region as a function of energy and redshift in Fig. 1. It can be seen that the characteristic energy of proton $E_{p}^{*} \sim 6.3 \times 10^{9} \mathrm{GeV}$ should be found in Fig. 1 as the point where the distance to the source is equal to the mean free path of protons at the present epoch $(z=0)$. The above issues give a strong constraint on the range of energy of the protons from $E_{\mathrm{p}, \min }=E_{\mathrm{p} \text {,low }}$ to $E_{\mathrm{p}, \max }=\min \left[E_{\mathrm{p}}^{*}, E_{\mathrm{GZK}}\right]$.

\section{Apply to 1 ES $0229+200$}

1ES 0229+200 resides in an elliptical host galaxy at a redshift of $z=0.1396$ (Woo et al. 2005). The source has been classified as a high-frequency peaked BL BLac object (HBL) due to its X-ray to radio flux ratio (Giommi et al. 1995). As a special source, the spectrum of 1 ES $0229+200$ was measured extended up to $10 \mathrm{TeV}$ with a hard archival spectral index at VHE of 2.5 \pm 0.19 (Aharonian et al. 2007; Aliu et al. 2014). Compiling with the VERITAS measured spectrum averaged over all three
Table 1. Linear regression analysis of the observed spectrum of 1ES $0229+200$ in the MeV-TeV energy band.

\begin{tabular}{lcc}
\hline \hline Photon energy & $\mathrm{MeV}-\mathrm{GeV}$ & $\gtrsim 1 \mathrm{TeV}$ \\
\hline$\Gamma(\sigma)$ & $1.93 \pm 0.06$ & $2.27 \pm 0.13$ \\
$r$ & 0.99 & 0.98 \\
$N$ & 13 & 13 \\
$p$ & $<0.0001$ & $<0.0001$ \\
\hline
\end{tabular}

Notes. The linear regression is obtained by considering the photon energy $E_{\gamma}$ to be the independent variable and assuming a relation $\mathrm{d} N_{\gamma}\left(E_{\gamma}\right) /$ $\mathrm{d} E_{\gamma} \propto E_{\gamma}^{-\Gamma} ; N$ is the number of points, $r$ is the correlation coefficient, and $p$ is the chance probability.

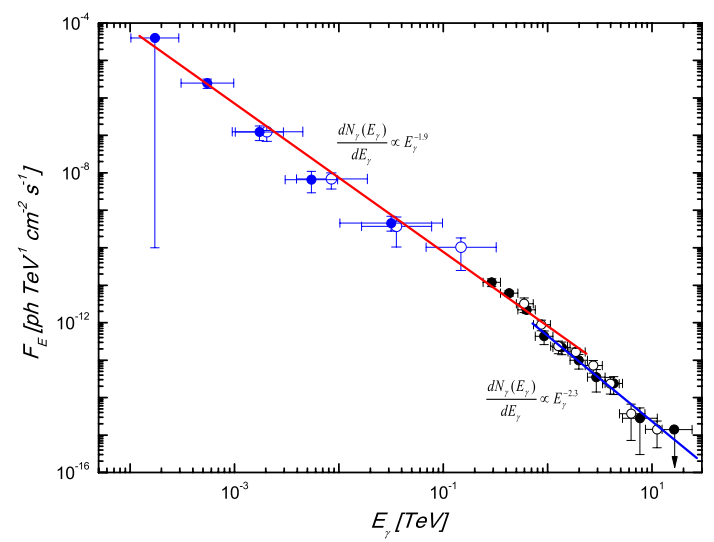

Fig. 2. Observed photon spectrum of 1ES $0229+200$. The solid lines are the linear regression results in the $\mathrm{MeV}-\mathrm{GeV}$ and $\mathrm{TeV}$ energy bands. The Fermi-LAT observed data come from the second catalog (blue open circle) and third catalog (blue solid circle). The HESS observed data (black open circle) come from Aharonian et al. (2007) and VERITAS observed data (black solid circle) come from Aliu et al. (2014).

seasons (Aliu et al. 2014), and four years of Fermi-LAT observed results ${ }^{2}$, we reanalyze the spectrum of 1 ES $0229+200$ assuming a relation $\mathrm{d} N_{\gamma}\left(E_{\gamma}\right) / \mathrm{d} E_{\gamma} \propto E_{\gamma}^{-\Gamma}$ in Fig. 2. The results of linear regression analysis are listed in Table 1 . It can be seen that the spectrum should be fitted by a power-law relation with the spectra index $\Gamma_{\mathrm{MeV}-\mathrm{GeV} \text {,obs. }}=1.93 \pm 0.06$ in the $\mathrm{MeV}-\mathrm{GeV}$ energy band, and $\Gamma_{\mathrm{TeV} \text {,obs. }}=2.27 \pm 0.13$ in the TeV energy band. Now that the model argues that the $\mathrm{TeV}$ emission in distant $\mathrm{TeV}$ blazars is dominated by two components, the external TeV photons could significantly repair the EBL attenuation, and leave a hard spectra to $100 \mathrm{TeV}$ energy band. Using the model in Sect. 2, we should expect to reproduce the hard $\mathrm{TeV} \gamma$-rays spectra.

In order to do so, first we search for the internal $\gamma$-ray component in the one-zone homogeneous SSC frame. Assuming the density normalization $K_{0}=5.57 \times 10^{3} \mathrm{erg}^{-1} \mathrm{~cm}^{-3}$, we calculate the high-energy electron distribution with a broken power law between $E_{\mathrm{e}, \text { in, } \text { min }}=4.34 \mathrm{GeV}$ and $E_{\mathrm{e}, \text { in,cut }}=4.5 \times 10^{4} \mathrm{GeV}$ with a break at $E_{\mathrm{e}, \mathrm{in}, \mathrm{b}}=4.49 \times 10^{2} \mathrm{GeV}$, where the energy index of the particles between $E_{\mathrm{e}, \text { in,min }}$ and $E_{\mathrm{e} \text {,in,b }}$ is set to $n_{1}=2.2$ and the energy index of the particles between $E_{\mathrm{e}, \text { in,b }}$ and $E_{\mathrm{e}, \text { in,cut }}$ is set to $n_{2}=4.0$, The parameters are used as follows: the magnetic field strength is $B=0.06 \mathrm{G}$, the emission region size is $R_{\mathrm{e}}=2.77 \times 10^{16} \mathrm{~cm}$, and the Doppler factor is $\delta=10.7$. We assume that relativistic electrons are in the steady state during

2 http://fermi.gsfc.nasa.gov/ssc/data/access/lat/ 4yr_catalog/ 


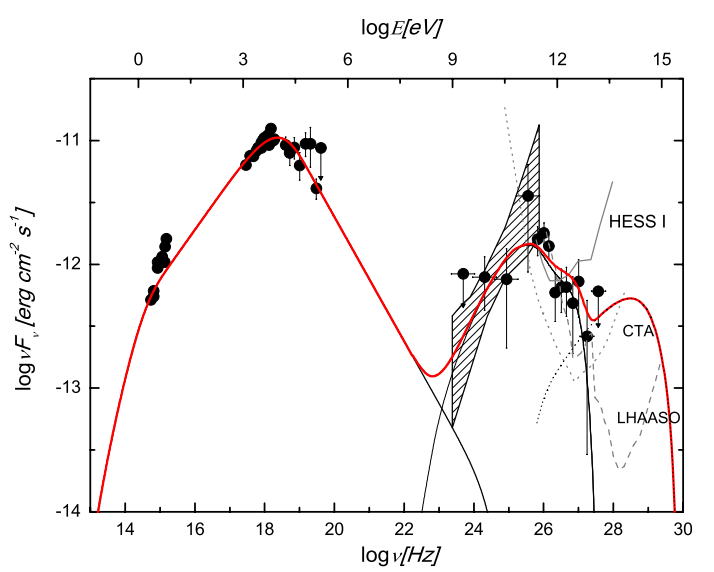

Fig. 3. Multiwavelength SED of the distant TeV blazar 1ES 0229+200. The solid black curve represents the internal component spectra with the absorption optical depth that is deduced by the LLL EBL model in Dwek \& Krennrich (2005), and the dotted black curve represents the external component spectra. The superposed model spectrum is plotted as a red curve. The observed data come from Aliu et al. (2014) (solid circle). The shaded area shows the Fermi upper bounds at the $99 \%$ confidence level. The differential sensitivity curve for the 50-h observation with HESS I ${ }^{3}$, gray solid curve, the 50-h differential sensitivity goal of the CTA (Actis et al. 2011, gray dashed curve), and the differential sensitivity curve for the one-year observation with LHAASO (Cui et al. 2014, gray dotted curve) are also shown.

the observational epoch. Therefore, we can calculate the TeV $\gamma$-ray spectrum in the one-zone homogeneous SSC frame using the broken power-law electron spectrum.

We now consider the external $\mathrm{TeV}$ photons production along the line of sight. We argue that the sites of electron acceleration may also accelerate protons in the jet. If the acceleration site is full of electrically neutral nonthermal plasma, the protonto-electron ratio depends on the spectral indices of injection electron and proton spectra (e.g., Schlickeiser 2002; Persic \& Rephaeli 2014). As a simple case, we do not specify the protonto-electron ratio and leave it as a free parameter with $L_{\mathrm{p}}$ and $L_{\mathrm{e}}$. Assuming the protons are accelerated to extra-relativistic energy in the acceleration region through some mechanisms and then escape from the region without energy loss. We calculate the Bethe-Heitler pairs distribution along the line of sight with a proton injection spectrum between $E_{\mathrm{p} \text {,min }}$ and $E_{\mathrm{p} \text {,max }}$, where, in this case, the correlation length of the random fields is set to $l_{\mathrm{c}}=1 \mathrm{Mpc}$, the IGMF is set to $B_{\mathrm{IG}}=10^{-15} \mathrm{G}$ (e.g., Tavecchio et al. 2010), and the power-law exponent $\alpha=2.0$ is adopted by fitting to the ultra high-energy cosmic rays data at the lower energies (Berezinsky et al. 2006). Since the typical luminosity range of AGNs are in $10^{45}-10^{47} \mathrm{erg} \mathrm{s}^{-1}$ (e.g., Ghisellini et al. 2014), in our calculation we set the jet power of protons $L_{\mathrm{p}}=0.3 \times 10^{46} \mathrm{erg} \mathrm{s}^{-1}$. We assume that the energy of the Bethe-Heitler pairs is lost through interaction with the CMB photons at the cosmological distance D during the observational epoch. Therefore, we can calculate the external TeV $\gamma$-ray spectrum along the line of sight using the injection electron spectrum.

We show the predicted internal component spectrum, external component spectrum, and the superposed model spectral in Fig. 3. For comparison, the differential sensitivity curve for the 50-h observation with HESS $\mathrm{I}^{3}$, the 50-h differential

\footnotetext{
3 http://www.mpi-hd.mpg.de/hfm/HESS/pages/home/ proposals/
}

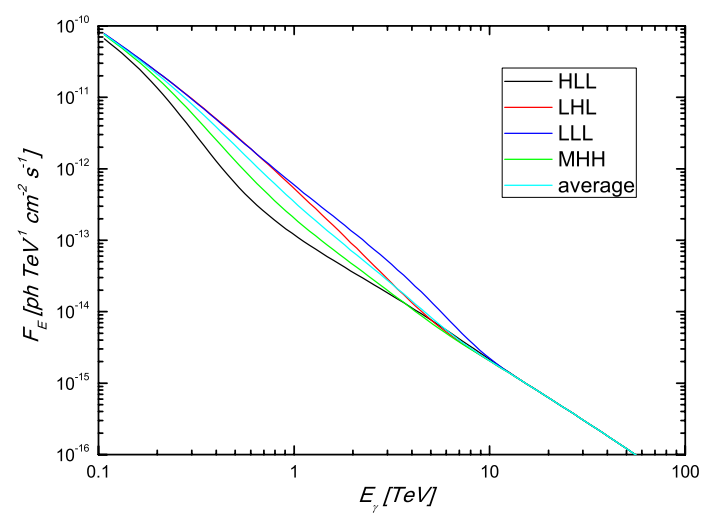

Fig. 4. Model spectra with different EBL absorption optical depths. It is indicated that, dependent of the EBL absorption optical depth, the photon spectra with the photon energy between $0.1 \mathrm{TeV}$ and $10 \mathrm{TeV}$ could be described by a relation $\mathrm{d} N_{\gamma}\left(E_{\gamma}\right) / \mathrm{d} E_{\gamma} \propto E_{\gamma}^{-\Gamma}$ with a small photon spectral index $1.5<\Gamma<2.5$, and even flatter in a higher energy band.

Table 2. Physical parameters of the model spectra.

\begin{tabular}{lllll}
\hline \hline \multicolumn{2}{c}{ Internal component } & & \multicolumn{2}{c}{ External component } \\
\cline { 1 - 2 } Parameters & Value & & Parameters & Value \\
\hline$K_{0}\left[\mathrm{erg}^{-1} \mathrm{~cm}^{-1}\right]$ & $5.57 \times 10^{3}$ & & $E_{\mathrm{p}, \max }[\mathrm{GeV}]$ & $6.3 \times 10^{9}$ \\
$n_{1}$ & 2.2 & & $\alpha$ & 2.0 \\
$n_{2}$ & 4.0 & & $B_{\mathrm{IG}}[\mathrm{G}]$ & $1.0 \times 10^{-15}$ \\
$E_{\mathrm{e}, \mathrm{in}, \min }[\mathrm{GeV}]$ & 4.34 & & $l_{\mathrm{c}}[\mathrm{Mpc}]$ & 1.0 \\
$E_{\mathrm{e}, \mathrm{in}, \mathrm{b}}[\mathrm{GeV}]$ & 449 & & $L_{\mathrm{p}}\left[\mathrm{erg} \mathrm{s}{ }^{-1}\right]$ & $0.3 \times 10^{46}$ \\
$E_{\mathrm{e}, \mathrm{in}, \mathrm{cut}}[\mathrm{GeV}]$ & $4.5 \times 10^{4}$ & & $D[\mathrm{Gpc}]$ & 1.0 \\
$B[\mathrm{G}]$ & 0.06 & & - & - \\
$\delta$ & 10.7 & & - & - \\
$R_{\mathrm{e}}[\mathrm{cm}]$ & $2.77 \times 10^{16}$ & & - & - \\
\hline
\end{tabular}

sensitivity goal of the Cherenkov Telescope Array (CTA, Actis et al. 2011), the differential sensitivity curve for the one-year observation with the Large High Altitude Air Shower Observatory (LHAASO, Cui et al. 2014), and the multi-wavelength observed data of 1ES 0229+200 (Aliu et al. 2014) are also shown. All of the physical parameters of the internal component and external component spectra are listed in Table 2. It can be seen that 1) the observed spectrum properties of 1ES 0229+200, especially the $\mathrm{TeV} \gamma$-ray tail of the observed spectra, were reproduced in our model; 2) an expected $\mathrm{TeV} \gamma$-ray spectrum with photon energy $>1 \mathrm{TeV}$ of $1 \mathrm{ES} 0229+200$ should be comparable with the 50-h sensitivity goal of the CTA and the differential sensitivity curve for the one-year observation with the LHAASO.

In order to intensively examine the hard spectra properties of 1ES $0229+200$ in the TeV energy bands. We show the model spectra with different EBL absorption optical depths in Fig. 4. Now that the predicted spectral shape of external photons is not very sensitive to the variations in the proton spectrum index $\alpha$ (e.g., Essey et al. 2010), we do not take into account the effect of $\alpha$ on the model spectra. It is indicated that, dependent of the EBL absorption optical depth, the photon spectra with the photon energy between $0.1 \mathrm{TeV}$ and $10 \mathrm{TeV}$ could be described by a relation $\mathrm{d} N_{\gamma}\left(E_{\gamma}\right) / \mathrm{d} E_{\gamma} \propto E_{\gamma}^{-\Gamma}$ with a small photon spectral index $1.5<\Gamma<2.5$, and even flatter in a higher energy band. As a special case with the LLL EBL model in Dwek \& Krennrich (2005), in Fig. 5 we compare the predicted differential photon 


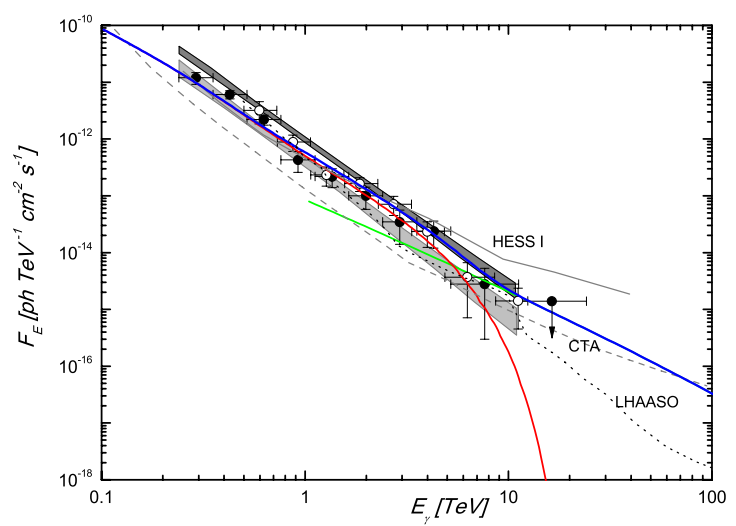

Fig. 5. Comparisons of predicted $\mathrm{TeV}$ photon spectra with observed data of the distant TeV blazar 1ES 0229+200. The upper and lower shaded regions show the spectral shape during the flaring and low periods. The red curve represents the internal component spectra with the absorption optical depth that is deduced by the LLL EBL model in Dwek \& Krennrich (2005), and the green curve represents external component spectra. The superposed model spectrum is plotted as a blue curve. The HESS Observed data (open circle) come from Aharonian et al. (2007) and VERITAS observed data (solid circle) come from Aliu et al. (2014). The integral sensitivity curve for the 50-h observation with HESS I $^{3}$, gray solid curve, the 50-h integral sensitivity goal of the CTA (Actis et al. 2011, gray dashed curve), and the integral sensitivity curve for the one-year observation with LHAASO (Cui et al. 2014, gray dotted curve) are also shown.

spectrum in the $\gtrsim 1 \mathrm{TeV} \gamma$-ray energy bands (blue solid curve) with observed data of 1ES $0229+200$ at HESS (Aharonian et al. 2007) and VERITAS (Aliu et al. 2014) energy bands. Our result shows that in this special case, superposed model spectra with the photon energy between $1 \mathrm{TeV}$ and $10 \mathrm{TeV}$ could be described by a photon spectral index $\Gamma=2.4$. This is in agreement with the result that is found by reanalysis of the spectrum of $1 \mathrm{ES} 0229+200$.

\section{Discussion and conclusions}

As an open issue, VHE $\gamma$-ray measurements of distant $\mathrm{TeV}$ blazars can be explained by $\mathrm{TeV}$ spectra induced by ultra highenergy cosmic rays (Essey \& Kusenko 2010; Essey et al. 2010; 2011b; Murase 2012; Murase et al. 2012; Razzaque et al. 2012; Takami et al. 2013; Zheng, et al. 2013). In this paper, We develop a model for a possible $\mathrm{TeV}$ emission in distant $\mathrm{TeV}$ blazars. The aim of the present work is to study in greater detail the contribution of pairs injected by the pe process along the line of sight to the $\mathrm{TeV}$ spectra in distant blazars. In the model, the $\mathrm{TeV}$ emission in distant $\mathrm{TeV}$ blazars is dominated by two mixed components: the first is the internal component where the photon energy around $1 \mathrm{TeV}$ is produced by IC scattering of the relativistic electrons on the synchrotron photons (SSC) with a correction for EBL absorbtion, and the second is the external component where the photon energy more than $1 \mathrm{TeV}$ is produced by the cascade emission from high-energy protons propagating through intergalactic space. Assuming a suitable model parameters, we apply the model to observed spectra of distant TeV blazars of $1 E S 0229+200$. Our results show that 1 ) the observed spectrum properties of 1ES $0229+200$, especially the $\mathrm{TeV} \gamma$-ray tail of the observed spectra, could be reproduced in our model and 2) an expected $\mathrm{TeV} \gamma$-ray spectrum with photon energy $>1 \mathrm{TeV}$ of $1 \mathrm{ES} 0229+200$ should be comparable with the 50-h sensitivity goal of the CTA and the differential sensitivity curve for the one-year observation with LHAASO. We argue that strong evidence for the Bethe-Heitler cascades along the line of sight as a plausible origin of hard spectra in distant TeV blazars could be obtained from VHE observations with CTA and LHAASO.

The present work differs from the earlier studies that assume that the pairs cascade process induced by ultra high-energy cosmic rays occurs at the source (e.g., Murase 2012; Murase et al. 2012; Petropoulou \& Mastichiadis 2015). We concentrate on the protons with energy below the GZK cutoff, which would propagate through cosmological distances. We argue that the outflows of the jets from AGNs are likely to contain coherent magnetic fields aligned with the jet, so that the accelerated protons remain in the scope of the initial jet rather than getting deflected. Since the pe process takes place outside the galaxy clusters of both the observer and the source, the cluster magnetic fields are irrelevant to this issue. Although we expect larger fields in the filaments and wall, only the IGMF present deep in the voids along the line of sight is important (Essey \& Kusenko 2010). Within the host galaxy, the propagated directions of the protons could be changed by the galactic magnetic fields, the broadening of the image due to deflections in it should less than $\Delta \theta_{\max } \sim r / D_{\text {source }}$ (Essey et al. 2010), where $r$ is the size of the host galaxy, and $D_{\text {source }}$ is the distance to the host galaxy. Furthermore, the possible thin walls of magnetic fields that might intersect the line of sight could not cause a deflection of more than $\Delta \theta \sim h / D_{\text {wall }}$ (Essey et al. 2010), where $h$ is the wall thickness and $D_{\text {wall }}$ is the distance to the wall. The model also does not take into account both the $\gamma$-ray photon spectrum and the pairs cascade from the decay of pions $\pi^{0}, \pi^{+}$, and $\pi^{-}$. We argue that the characteristic energy of both decay induced photons $E_{\gamma} \sim 0.1 E_{\mathrm{p}}>10^{3} \mathrm{TeV}$ and pairs cascade from the decay of pions induced photons $E_{\gamma} \sim$ $\left(0.05 E_{\mathrm{p}} / m_{\mathrm{e}} c^{2}\right)^{2} E_{\mathrm{CMB}}>10^{4} \mathrm{TeV}$ with IGMF $B_{\mathrm{IG}}=10^{-15} \mathrm{G}$ and correlation length of the random fields $l_{\mathrm{c}}=1 \mathrm{Mpc}$. This energy is far from the TeV energy band.

It is noted that small photon indices are not easy to achieve in traditional leptonic scenarios, although the stochastic acceleration model (Lefa et al. 2011) and the leptohadronic model (Cerruti et al. 2015) can also explain the spectral hardening of $\mathrm{TeV}$ blazars because radiative cooling tends to produce particle energy distributions that are always steeper than $E^{-2}$. The above distribution results in a TeV photon index $\Gamma_{\mathrm{TeV} \text {,int }} \geq 1.5$, and even steeper at VHE due to the suppression of the Klein-Nishina effects of the cross-section (e.g., Chiang \& Böttcher, 2002). Even when the absorption effect by the lowest level EBL is used, the emitted spectra still tend to be steeper with an observed photon index $\Gamma_{\mathrm{TeV} \text {,obs }} \geq 2.5$ (e.g., Aharonian et al. 2007; Dwek \& Krennrich 2012). Because the Bethe-Heitler pair-creation rate is smoothed in the model, we argue that the spectral shape of the external component spectrum is not very sensitive to the proton injection spectrum (Essey et al. 2010); it is determined primarily by the spectrum of the CMB photons and the Bethe-Heitler pairs energy loss process, which result in hard $\mathrm{TeV}$ photons spectra.

Alternatively, the secondary $e^{ \pm}$pairs that are produced by $\gamma+\gamma \rightarrow e^{+}+e^{-}$pair creation generate a new $\gamma$-ray component through IC scattering of these $e^{ \pm}$pairs against target photons of the $\mathrm{CMB}$, initiating an electromagnetic cascade if the produced $\gamma$ ray is subsequently absorbed (e.g., Dai et al. 2002; Fan et al. 2004; Yang et al. 2008; Neronov et al. 2012). In order to reproduce a hard spectra, we include an external $\gamma$-ray component with photon energy around $\sim 10-100 \mathrm{TeV}$. Using the photon energy of the external $\gamma$-ray component, we 
could estimate the boosting energy through the IC process of $E_{\gamma} \sim \gamma_{e^{ \pm}}^{2} \epsilon_{\mathrm{CMB}} \sim 0.01-1.0 \mathrm{TeV}$. In this view, the resultant secondary photons should contribute to the $\mathrm{TeV} \gamma$-ray flux and we expect to find a complex spectrum around $1 \mathrm{TeV}$. However, ultra high-energy cosmic rays with $E_{\mathrm{p}} \sim 10^{19} \mathrm{eV}$ have energy loss paths $\lambda_{\mathrm{p} \gamma, \mathrm{e}} \sim 1 \mathrm{Gpc}$ for Bethe-Heitler pair production, whereas 10-100 TeV $\gamma$-ray only travel $\lambda_{\gamma \text {,eff }} \sim 3-200 \mathrm{Mpc}$ before being absorbed by $\gamma \gamma$ pairs production. Now that we assume the cascade emission region $D \sim \lambda_{\mathrm{p} \gamma, \mathrm{e}}$, the source at a redshift of $z<1.0$ allows the Bethe-Heitler pair to be injected and cascade to such energy far from the source. Thus, some $\mathrm{TeV}$ photons can reach the Earth before being attenuated even when $\tau\left(E_{\gamma}, z\right) \gg 1$, without any spectral shape transformation (Takami et al. 2013). On the other hand, although we focus on spectral information to the possible $\mathrm{TeV}$ emission in distant blazars, variability is also another important clue. Murase et al. (2012) argue that the cascade components would not have short variability timescales, since the shortest time scales are $\sim 1.0\left(E_{\gamma} / 10 \mathrm{GeV}\right)^{-2}\left(B_{\mathrm{IG}} / 10^{-18} \mathrm{G}\right)^{2} \mathrm{yr}$ in the $\gamma$-ray induced cascade case, and $\sim 10\left(E_{\gamma} / 10 \mathrm{GeV}\right)^{-2}\left(B_{\mathrm{IG}} / 10^{-18} \mathrm{G}\right)^{2} \mathrm{yr}$ in the ultra high-energy cosmic ray induced cascade case. The above issue suggests that strong variability is possible in the $\gamma$-ray induced cascade case, and this means that the cascaded $\gamma$-ray should be regarded as a mixture of attenuated and cascade components, and then the cascade component could be suppressed. Instead, in the ultra high-energy cosmic ray induced cascade case, the variability could not be found (Takami et al. 2013). The lack of strong evidence of variability in 1ES 0229+200 above the HESS energy band (Aharonian et al. 2007; Aliu et al. 2014) suggests that the ultra high-energy cosmic ray induced cascade component might dominate on the $\mathrm{TeV} \gamma$-ray spectrum. Since, in our issue, the $\mathrm{TeV}$ emission in distant $\mathrm{TeV}$ blazars is dominated by two components, the external $\mathrm{TeV}$ photons could significantly repair the EBL attenuation, and leave a hard spectra to $100 \mathrm{TeV}$ energy band.

It is clear that the jet power of protons plays an important role in determining an emission intensity. In our results, in order to obtain the TeV emission of the 1ES 0229+200, we adopt the jet power of protons $L_{\mathrm{p}}=0.3 \times 10^{46} \mathrm{erg} \mathrm{s}^{-1}$. It is well known that the jets of AGN are powered by the accretion of matter onto a central black hole (e.g., Urry \& Padovani 1995). On the assumption that the radiation escapes isotropically from the black hole, the balancing of the gravitational and radiation force leads to the maximum possible luminosity due to accretion $L_{\text {edd }} \sim 1.26 \times 10^{38} M / M_{\odot} \operatorname{erg~s}^{-1}$ (e.g., Dermer \& Menon 2009). When the total emission of an AGN is not super-Eddington, the Eddington luminosity is the maximum power available for the two jets, $P_{\text {jet }} \leq L_{\text {edd }} / 2$. In this view, the required power in protons could easily be provided by the source with mass $M=1.44 \times 10^{9} M_{\odot}($ Wagner 2008).

A potential drawback of the model is that the shape of the model spectra in the $1-10 \mathrm{TeV}$ energy ranges strongly depends on the level of the EBL. As a check, we constrain the shape of the model of spectra depending on a general EBL model. On the basis of the model results, we argue that the predicted $\mathrm{TeV}$ spectra properties of the above-mentioned model should be testable in the near future since the secondary emission process will expect CTA to detect more than $80 \mathrm{TeV}$ blazars in the above $1 \mathrm{TeV}$ energy band (Inoue et al. 2013). We note that the neutrino populations could be expected in $p \gamma$ interactions, and it should be given a clearer predictive character with the IceCube observations. Unfortunately, the pe process does not contain any neutrino populations. We defer this possibility to future work. Although our model focuses on the contribution of pairs injected by pe process along the line of sight to the TeV spectra, the $\gamma$-ray induced cascade is also another important scenario for the possible $\mathrm{TeV}$ emission in distant blazars (e.g., Vovk et al. 2012; Takami et al. 2013). We leave this possibility to the observation of CTA (Actis et al. 2011), LHAASO (Cao 2010; Cui et al. 2014), HAWC (Sandoval et al. 2009), and HiSCORE (Hampf et al. 2011).

Acknowledgements. We thank the anonymous referee for valuable comments and suggestions. This work is partially supported by the National Natural Science Foundation of China under grants 11463007, U1231203, Science and Technology in support of Yunnan Province Talent under grants 2012HB014, and the Natural Science Foundation of Yunnan Province under grant 2013FD014. This work is also supported by the Science Foundation of Yunnan educational department (grant 2012Z016).

\section{References}

Abramowski, A., Acero, F., Aharonian, F., et al. 2013, A\&A, 550, A4 Acciari, V. A., Aliu, E., Arlen, T., et al. 2009, ApJ, 693, L104 Ackermann, M., Ajello, M., Allafort, A., et al. 2012, Science, 338, 1190

Actis, M., Agnetta, G., Aharonian, F., et al. 2011, Exp. Astron., 32, 193 Aharonian F. A. 2000, New Astron., 5, 377

Aharonian, F., Akhperjanian, A. G., Bazer-Bachi, A. R., et al. 2006a, Nature, 440,1081

Aharonian, F., Akhperjanian, A. G., Bazer-Bachi, A. R., et al. 2006b, Science, 314,1424

Aharonian, F., Akhperjanian, A. G., Barres de Almeida, U., et al. 2007, A\&A, 475, L9

Aharonian, F. A., Kelner, S. R., \& Prosekin, A. 2010, Phy. Rev. D, 82, 043002

Aharonian, F., Essey, W., Kusenko, A., \& Prosekin, A. 2013, Phy. Rev. D, 87, 063002

Aliu, E., Archambault, S., Arlen, T., et al. 2014, ApJ, 782, 13

Atoyan, A., \& Dermer, C. D. 2001, Phys. Rev. Lett., 87, 221102

Berezinsky, V., Gazizov, A. Z., \& Grigorieva, S. I. 2006, Phy. Rev. D, 74, 043005

Bloom S. D., \& Marscher A. P. 1996, ApJ, 461, 657

Blumenthal, G. R. 1970, Phys. Rev. D, 1, 1596

Böttcher, M., \& Dermer, C. D. 1998, ApJ, 501, L51

Cao, Z. 2010, Chin. Phys. C, 34, 249

Cerruti, M., Zech, A., Boisson, C., \& Inoue, S. 2015, MNRAS, 448, 910

Chiang, J., \& Böttcher, M. 2002, ApJ, 564, 92

Chodorowski, M. J., Zdziarski, A. A., \& Sikora, M. 1992, ApJ, 400, 181

Costamante, L. 2013, Inter. J. Mod. Phys. D, 22, 30025

Costamante, L., Ghisellini, G., Giommi, P., et al. 2001, A\&A, 371, 512

Costamante, L., Aharonian, F., Buhler, R., et al. 2008, in High Energy Gammaray Astronomy, eds. F. A. Aharonian, W. Hofmann, \& E. Rieger (Melville, NY: AIP), AIP Conf. Proc., 1085, 644

Cui, S. W., Liu, Y., Liu, Y. J., et al. 2014, Astropart. Phys., 54, 86

Dai, Z. G., Zhang, B., Gou, L. J., et al. 2002, ApJ, 580, L7

de Angelis, A., Roncadelli, M., \& Mansutti, O. 2007, Phy. Rev. D, 76, 121301

Dermer, C. D., \& Menon, G. 2009, High Energy Radiation from Black Holes: Gamma Rays, Cosmic Rays, and Neutrinos

Dermer, C. D., \& Schlickeiser, R. 1993, ApJ, 416, 458

Dermer, C. D., Schlickeiser, R., \& Mastichiadis, A. 1992, A\&A, 256, L27

Dermer, C. D., Razzapue, S., Finke, J. D., \& Atoyan, A. 2009, New J. Phys., 11, 065016

Dimitrakoudis, S., Mastichiadis, A., Protheroe, R. J., \& Reimer, A. 2012, A\&A, 546, A120

Dwek, E., \& Krennrich, F. 2005, ApJ, 618, 657

Dwek, E., \& Krennrich, F. 2013, Astropart. Phys., 43, 112

Essey, W., \& Kusenko, A. 2010, Astropart. Phys., 33, 81

Essey, W., Kalashev, O. E., Kusenko, A., \& Beacom, J. F. 2010, Phys. Rev. Lett., 104,141102

Essey, W., Ando, S., Kusenko, A. 2011a, Astropart. Phys., 35, 135

Essey, W., Kalashev, O. E., Kusenko, A., \& Beacom, J. F. 2011b, ApJ, 731, 51

Fan, Y. Z., Dai, Z. G., \& Wei, D. M. 2004, A\&A, 415, 483

Finke, J. D., \& Razzaque, S. 2009, ApJ, 698, 1761

Franceschini, A., Rodighiero, G., \& Vaccari, M. 2008, A\&A, 487, 837

Ghisellini, G., \& Madau, P. 1996, MNRAS, 280, 67

Ghisellini, G., Tavecchio, F., Maraschi, L., et al. 2014, Nature, 515, 376

Giommi, P., Ansari, S. G., \& Micol, A. 1995, A\&AS, 109, 267

Gould, R., \& Schreder, G. 1966, Phys. Rev. Lett., 16, 252

Greisen, K. 1966, Phys. Rev. Lett., 16, 748

Hampf, D., Tluczykont, M., \& Horns, D. 2011, Proc. of TEXAS 2010 Conf. in Heidelberg [arXiv: 1104.2336] 
Inoue, Y., Kalashev, O. E., \& Kusenko, A. 2013, Astropart. Phys., 54, 118 Kalashev, O. E., Kusenko, A., \& Essey, W. 2013, Phys. Rev. Lett., 111, 041103 Katarzynski, K., Sol, H., \& Kus, A. 2001, A\&A, 367, 809

Kifune, T. 1999, ApJ, 518, L21

Kneiske, T. M., Bretz, T., Mannheim, K., \& Hartmann, D. H. 2004, A\&A, 413, 807

Konopelko, A., Mastichiadis, A., Kirk, J., de Jager, O. C., \& Stecker, F. W. 2003, ApJ, 597, 851

Lefa, E., Rieger, F. M., \& Aharonian, F. 2011, ApJ, 740, L64

Mannheim, K. 1993, A\&A, 269, 67

Mannheim, K., \& Biermann, P. L. 1992, A\&A, 253, L21

Maraschi, L., Ghisellini, G., \& Celotti, A. 1992, ApJ, 397, L5

Mastichiadis, A., \& Kirk, J. G. 1995, A\&A, 295, 613

Mastichiadis, A., \& Kirk, J. G. 1997, A\&A, 320, 19

Mastichiadis, A., Protheroe, R. J., \& Kirk, J. G. 2005, A\&A, 433, 765

Maximon, L. C. 1968, J. Res. Natl. Bur. Std., 72, 79

Mazin, D., \& Raue, M. 2007, A\&A, 471, 439

Mücke, A., \& Protheroe, R. 2001, Astropart. Phys., 15, 121

Mücke, A., Protheroe, R. J., Engel, R., Rachen, J. P., \& Stanev, T. 2003, Astropart. Phys., 18, 593

Murase, K. 2012, ApJ, 745, L16

Murase, K., \& Takami, H. 2009, ApJ, 690, L14

Murase, K., Dermer, C. D., Takami, H., \& Migliori, G. 2012, ApJ, 749, 63

Neronov, A., Semikoz, D., \& Taylor, A. M. 2012, A\&A, 541, A31

Nikishov, A. 1962, J. Exp. Theor. Phys., 14, 393

Persic, M., \& Rephaeli, Y. 2014, A\&A, 567, A101

Petropoulou, M. 2014, MNRAS, 442, 3026

Petropoulou, M., \& Mastichiadis, A. 2015, MNRAS, 447, 36

Pohl, M., \& Schlickeiser, R. 2000, A\&A, 354, 395
Protheroe, R. J., \& Meyer, H. 2000, Phys. Lett. B, 493, 1

Raiteri, C. M., Villata, M., Smith, P. S., et al. 2012, A\&A, 545, A48

Romero, G. E., \& Vila, G. S. 2008, A\&A, 485, 623

Sanchez, D. A., Fegan, S., \& Giebels, B. 2013, A\&A, 554, A75

Sanchez-Conde, M. A., Paneque, D., Bloom, E., Prada, F., \& Dominguez, A. 2009, Phy. Rev. D, 79, 123511

Sandoval, A., Alfaro, R., Belmont, E., et al. 2009, Fermi Symp., eConf Proc. C091122 [arXiv:0912.3329]

Schlickeiser, R. 2002, Cosmic Ray Astrophysics (Berlin: Springer), 472

Sikora, M., Begelman, M. C., \& Rees, M. J. 1994, ApJ, 421, 153

Simet, M., Hooper, D., \& Serpico, P. D. 2008, Phy. Rev. D, 77, 063001

Stecker, F. W., de Jager, O. C., \& Salamon, M. H. 1992, ApJ, 390, L49

Stecker, F. W., Malkan, M. A., \& Scully, S. T. 2006, ApJ, 648, 774

Sobolewska, M. A., Siemiginowska A., Kelly, B. C., \& Nalewajko, K. 2014, ApJ, 786, 143

Takami, H., Murase, K., \& Dermer, C. D. 2013, ApJ, 771, L32

Tavecchio, F., Ghisellini, G., Foschini, L., et al. 2010, MNRAS, 406, L70

Urry, C. M. 1998, Adv. Space Res., 21, 89

Urry, C. M., \& Padovani, P. 1995, PASP, 107, 803

Venters, T. M. 2010, ApJ, 710, 1530

Vovk, I., Taylor, A. M., Semikoz, D., \& Neronov, A. 2012, ApJ, 747, L14

Wagner, R. M. 2008, MNRAS, 385, 119

Woo, J. H., Urry, C. M., van der Marel, R. P., Lira, P., \& Maza, J. 2005, ApJ, 631,762

Wu, Z. Z., Jiang, D. R., Gu, M. F., \& Liu, Y. 2007, A\&A, 466, 63

Yang, C. Y., Fang, J., Lin, G. F., \& Zhang, L. 2008, ApJ, 682, 767

Zatsepin, G. T., \& Kuzmin, V. A. 1966, J. Exp. Theor. Phys. Lett., 4, 78

Zheng, Y. G., \& Kang, T. 2013, ApJ, 764, 113

Zheng, Y. G., \& Zhang, L. 2011, ApJ, 728, 105 